\title{
Kinematic Self-Similar Plane Symmetric Solutions
}

\author{
M. Sharif *and Sehar Aziz ${ }^{\dagger}$ \\ Department of Mathematics, University of the Punjab, \\ Quaid-e-Azam Campus, Lahore-54590, Pakistan.
}

\begin{abstract}
This paper is devoted to classify the most general plane symmetric spacetimes according to kinematic self-similar perfect fluid and dust solutions. We provide a classification of the kinematic self-similarity of the first, second, zeroth and infinite kinds with different equations of state, where the self-similar vector is not only tilted but also orthogonal and parallel to the fluid flow. This scheme of classification yields twenty four plane symmetric kinematic self-similar solutions. Some of these solutions turn out to be vacuum. These solutions can be matched with the already classified plane symmetric solutions under particular coordinate transformations. As a result, these reduce to sixteen independent plane symmetric kinematic self-similar solutions.
\end{abstract}

PACS: 04.20.-q, 04.20.Jb

Keywords: Plane symmetry, Self-similar variable.

\section{Introduction}

Einstein field equations (EFEs) are highly non-linear, second order coupled partial differential equations and hence could not be solved unless certain symmetry assumptions are taken on the spacetime metric. There has been

*msharif@math.pu.edu.pk

†'sehar_aziz@yahoo.com 
a recent literature [1-7, and references therein] which shows a significant interest in the study of various symmetries. Self-similarity leads to ordinary differential equations (ODEs) and their mathematical treatment is relatively simple. Invariance of the field equations under a scale transformation indicates that there exist scale invariant solutions to the EFEs. These solutions are known as self-similar solutions. Although self-similar solutions are only special solutions, they often play an important role in cosmological situations and gravitational collapse.

There exist several preferred geometric structures in self-similar models and a number of natural approaches may be used in studying them. The three most common ones are the co-moving, homothetic and Schwarzschild approaches. Each of the approaches has its individual physical interpretational advantages and they are all complementary. In the co-moving approach, pioneered by Cahill and Taub [8], the coordinates are adopted to the fluid 4-velocity vector. This probably affords the best physical insight and is the most convenient one. In General Relativity (GR), a self-similarity defined by the existence of a homothetic vector (HV) field is called self-similarity of the first kind (or homothety or continuous self similarity). There exists a natural generalization of homothety called kinematic self-similarity, which is defined by the existence of a kinematic self-similar (KSS) vector field.

Cahill and Taub [8] gave the concept of self-similarity corresponding to Newtonian self-similarity of the homothetic class. Carter and Henriksen $[9,10]$ defined self-similarity of the second, zeroth and infinite kinds. The only compatible barotropic equation of state with self-similarity of the first kind is $p=k \rho$. The classification of the self-similar perfect fluid solutions of the first kind in the dust case $(k=0)$ has been provided by Carr [1]. The case $0<k<1$ has been studied by Carr and Coley [2]. Coley [11] has shown that the FRW solution is the only spherically symmetric homothetic perfect fluid solution in the parallel case. McIntosh [12] has discussed that a stiff fluid $(k=1)$ is the only compatible perfect fluid with the homothety in the orthogonal case. Benoit and Coley [13] have studied analytic spherically symmetric solutions of the EFEs coupled with a perfect fluid and admitting a KSS vector of the first, second and zeroth kinds. Sintes et al. [14] have considered spherically, plane and hyperbolic symmetric spacetimes which admit a KSS vector of the infinite kind with perfect fluid. Carr et al. $[15,16]$ have explored the KSS vector associated with the critical behavior observed in the gravitational collapse of spherically symmetric perfect fluid with equation of state $p=k \rho$. Further, they have investigated solution space 
of self-similar spherically symmetric perfect fluid models and physical aspects of these solutions. Coley and Goliath [17] have investigated self-similar spherically symmetric cosmological models with a perfect fluid and a scalar field with an exponential potential.

The assumption of self-similarity is very powerful in finding analytical solutions. The group $G_{3}$ contains two special cases of physical interest, i.e., spherical and plane symmetry. Most of the literature is available on spherical symmetric spacetimes. Maeda et al. [3,4] have studied the KSS vector of the second, zeroth and infinite kinds in the tilted, parallel and orthogonal cases. The same authors [5] have also discussed the classification of the spherically symmetric KSS perfect fluid and dust solutions. This analysis has provided some interesting solutions. Recently, Sharif and Sehar [7] have investigated the KSS solutions for the cylindrically symmetric spacetimes. The analysis has been extensively given for the perfect fluid and dust cases with tilted, parallel and orthogonal vectors by using different equations of state. Some interesting consequences have been developed. The same authors have also studied the properties of such solutions for spherically symmetric [18], cylindrically symmetric [19] and plane symmetric spacetimes [20].

In a recent paper, Sharif and Sehar [21] have explored the KSS solutions for the plane symmetric spacetimes under certain assumption. The investigation is incomplete due to this restriction on plane symmetric spacetimes. In this paper, we drop this restriction and deal with the most general plane symmetric spacetimes. This analysis provides many more interesting selfsimilar solutions. The paper has been organised as follows. In section 2, we briefly review KSS vector of different kinds corresponding to the plane symmetric spacetimes. Sections 3 and 4 are devoted to the titled perfect fluid and dust solutions respectively. The orthogonal perfect fluid and dust solutions are investigated in section 5. Sections 6 and 7 are used to explore the parallel perfect fluid and dust cases respectively. In the last section, we present summary of the results and their discussion.

\section{Plane Symmetric Spacetime and Kinematic Self-Similarity}

A plane symmetric spacetime is a Lorentzian manifold possessing a physical stress-energy tensor. This admits $S O(2)\left(\times \Re^{2}\right.$ as the minimal isometry 
group in such a way that the group orbits are spacelike surfaces of constant curvature. The most general plane symmetric metric is given in the form [22]

$$
d s^{2}=e^{2 \nu(t, x)} d t^{2}-e^{2 \mu(t, x)} d x^{2}-e^{2 \lambda(t, x)}\left(d y^{2}+d z^{2}\right),
$$

where $\nu, \mu$ and $\lambda$ are arbitrary functions of $t$ and $x$. The energy-momentum tensor for a perfect fluid can be written as

$$
T_{a b}=[\rho(t, x)+p(t, x)] u_{a} u_{b}-p(t, x) g_{a b}, \quad(a, b=0,1,2,3),
$$

where $\rho$ is the density, $p$ is the pressure and $u_{a}$ is the four-velocity of the fluid element. In the co-moving coordinate system, the four-velocity can be written as $u_{a}=\left(e^{\nu(t, x)}, 0,0,0\right)$. Using Eqs.(1) and (2), we can write the EFEs as

$$
\begin{aligned}
\kappa \rho & =e^{-2 \mu}\left(2 \lambda_{x} \mu_{x}-3 \lambda_{x}{ }^{2}-2 \lambda_{x x}\right)+e^{-2 \nu}\left(2 \lambda_{t} \mu_{t}+\lambda_{t}{ }^{2}\right), \\
0 & =\lambda_{t x}-\lambda_{t} \nu_{x}+\lambda_{t} \lambda_{x}-\lambda_{x} \mu_{t} \\
\kappa p & =e^{-2 \mu}\left(\lambda_{x}{ }^{2}+2 \lambda_{x} \nu_{x}\right)-e^{-2 \nu}\left(2 \lambda_{t t}-2 \lambda_{t} \nu_{t}+3 \lambda_{t}{ }^{2}\right), \\
\kappa p & =e^{-2 \mu}\left(\nu_{x x}+\nu_{x}{ }^{2}+\nu_{x} \lambda_{x}+\lambda_{x}{ }^{2}+\lambda_{x x}-\lambda_{x} \mu_{x}-\nu_{x} \mu_{x}\right) \\
& -e^{-2 \nu}\left(\lambda_{t t}-\lambda_{t} \nu_{t}+\lambda_{t}^{2}+\mu_{t t}+\mu_{t}^{2}+\lambda_{t} \mu_{t}-\nu_{t} \mu_{t}\right) .
\end{aligned}
$$

It follows from the conservation of energy-momentum tensor, $T_{; b}^{a b}=0$, that

$$
\mu_{t}=-\frac{\rho_{t}}{(\rho+p)}-2 \lambda_{t}
$$

and

$$
\nu_{x}=-\frac{p_{x}}{(\rho+p)} .
$$

For a plane symmetric spacetime, the general form of a vector field $\xi$ can be written as

$$
\xi^{a} \frac{\partial}{\partial x^{a}}=h_{1}(t, x) \frac{\partial}{\partial t}+h_{2}(t, x) \frac{\partial}{\partial x},
$$

where $h_{1}$ and $h_{2}$ are arbitrary functions. The vector field $\xi$ can have three cases, i.e., parallel, orthogonal and tilted. They are distinguished by the relation between the generator and a timelike vector field, which is identified as the fluid flow, if it exists. When $\xi$ is parallel to the fluid flow, $h_{2}=0$ and when $\xi$ is orthogonal to the fluid flow $h_{1}=0$. When both $h_{1}$ and $h_{2}$ are non-zero, $\xi$ is tilted to the fluid flow. The tilted case is the most general among them. 
We define a KSS vector $\xi$ such that

$$
\begin{aligned}
£_{\xi} h_{a b} & =2 \delta h_{a b}, \\
£_{\xi} u_{a} & =\alpha u_{a},
\end{aligned}
$$

where $h_{a b}=g_{a b}-u_{a} u_{b}$ is the projection tensor, $\alpha$ and $\delta$ are constants. The ratio, $\alpha / \delta$, is called the similarity index which gives rise to the following two cases:

$$
\text { 1. } \delta \neq 0 ; \quad 2 . \quad \delta=0
$$

Case 1: When $\delta \neq 0$, we can choose it as unity and the KSS vector for the titled case can take the following form

$$
\xi^{a} \frac{\partial}{\partial x^{a}}=(\alpha t+\beta) \frac{\partial}{\partial t}+x \frac{\partial}{\partial x} .
$$

In this case, the similarity index, $\alpha / \delta$, further implies the following three possibilities

(i) $\quad \delta \neq 0, \quad \alpha=1 \quad(\beta$ can be taken to be zero),

(ii) $\quad \delta \neq 0, \quad \alpha=0 \quad$ ( $\beta$ can be taken to be unity),

(iii) $\quad \delta \neq 0, \quad \alpha \neq 0,1 \quad(\beta$ can be taken to be zero).

The first case 1(i) is referred to the self-similarity of the first kind. In this case, $\xi$ is a homothetic vector and the self-similar variable $\xi$ turns out to be $x / t$. For the second case 1(ii), it is called the self-similarity of the zeroth kind and the self-similar variable becomes $\xi=x e^{-t}$. The last case 1(iii) is known as the self-similarity of the second kind and the self-similar variable turns out to be

$$
\xi=\frac{x}{(\alpha t)^{\frac{1}{\alpha}}} .
$$

For the case (1), the metric functions take the following form

$$
\nu=\nu(\xi), \quad \mu=\mu(\xi), \quad e^{\lambda}=x e^{\lambda(\xi)} .
$$

Case 2: In the second case (2), when $\delta=0$ and $\alpha \neq 0$ ( $\alpha$ can be unity and $\beta$ can be re-scaled to zero), the self-similarity is referred to the infinite kind. Here, the KSS vector $\xi$ becomes

$$
\xi^{a} \frac{\partial}{\partial x^{a}}=t \frac{\partial}{\partial t}+x \frac{\partial}{\partial x}
$$


and the self-similar variable takes the form $\xi=x / t$. Consequently, the metric functions will become

$$
\nu=\nu(\xi), \quad \mu=-\ln (x)+\mu(\xi), \quad \lambda=\lambda(\xi) .
$$

It is mentioned here that for $\delta=0=\alpha$, the KSS vector $\xi$ reduces to KV.

When the KSS vector $\xi$ is parallel to the fluid flow, we obtain

$$
\xi^{a} \frac{\partial}{\partial x^{a}}=f(t) \frac{\partial}{\partial t}
$$

where $f(t)$ is an arbitrary function. It is worth mentioning point here that we obtained [21] contradictory results in the first, second and zeroth kinds while for the infinite kind the self-similar variable was $x$. As a result, there was no solution when $\xi$ was parallel to the fluid flow in the first, second and zeroth kinds except for the infinite kind. However, this analysis of the most general plane symmetric spacetimes yields self-similar variable $x$ in each kind and hence we can expect solution. The metric functions for the first, second, zeroth and infinite kinds, respectively, will be

$$
\begin{aligned}
& \nu=\nu(x), \quad \mu=\ln (t)+\mu(x), \quad \lambda=\ln (t)+\lambda(x), \\
& \nu=(\alpha-1) \ln (t)+\nu(x), \quad \mu=\ln (t)+\mu(x), \quad \lambda=\ln (t)+\lambda(x), \\
& \nu=-\ln (t)+\nu(x), \quad \mu=\ln (t)+\mu(x), \quad \lambda=\ln (t)+\lambda(x), \\
& \nu=\nu(x), \quad \mu=\mu(x), \quad \lambda=\lambda(x) .
\end{aligned}
$$

If the KSS vector $\xi$ is orthogonal to the fluid flow, it follows that

$$
\xi^{a} \frac{\partial}{\partial x^{a}}=g(x) \frac{\partial}{\partial x}
$$

where $g(x)$ is an arbitrary function and the self-similar variable is $t$. The corresponding metric functions for the first, second, zeroth and infinite kinds, respectively, will take the following form

$$
\begin{aligned}
& \nu=\ln (x)+\nu(t), \quad \mu=\mu(t), \quad \lambda=\ln (x)+\lambda(t), \\
& \nu=\alpha \ln (x)+\nu(t), \quad \mu=\mu(t), \quad \lambda=\ln (x)+\lambda(t), \\
& \nu=\nu(t), \quad \mu=\mu(t), \quad \lambda=\ln (x)+\lambda(t), \\
& \nu=\ln (x)+\nu(t), \quad \mu=-\ln (x)+\mu(t), \quad \lambda=\lambda(t) .
\end{aligned}
$$


The following two types of polytropic equations of state (EOS) will be assumed. The first equation of state, denoted by $\operatorname{EOS}(1)$, is

$$
p=k \rho^{\gamma},
$$

where $k$ and $\gamma$ are constants. Another EOS is the following [17]

$$
p=k n^{\gamma}, \quad \rho=m_{b} n+\frac{p}{\gamma-1},
$$

where $m_{b}$ is a constant which corresponds to the baryon mass, and $n(t, r)$ corresponds to baryon number density. We call this equation as the second equation of state $\operatorname{EOS}(2)$. Notice that we take $k \neq 0$ and $\gamma \neq 0,1$ for $\operatorname{EOS}(1)$ and $\operatorname{EOS}(2) \cdot \operatorname{EOS}(3)$ is given by

$$
p=k \rho, \quad-1 \leq k \leq 1, \quad k \neq 0 .
$$

\section{$3 \quad$ Tilted Perfect Fluid Case}

\subsection{Self-Similarity of the First Kind}

It follows from the EFEs that the energy density $\rho$ and pressure $p$ must take the following form

$$
\begin{aligned}
\kappa \rho & =\frac{1}{x^{2}} \rho(\xi), \\
\kappa p & =\frac{1}{x^{2}} p(\xi),
\end{aligned}
$$

where the self-similar variable is $\xi=x / t$. When the EFEs and the equations

of motion for the matter field are satisfied, it yields a set of ODEs and hence Eqs.(3)-(8) reduce to

$$
\begin{aligned}
\dot{\rho} & =-(\dot{\mu}+2 \dot{\lambda})(\rho+p), \\
2 p-\dot{p} & =\dot{\nu}(\rho+p), \\
e^{2 \mu} \rho & =2 \dot{\mu}+2 \dot{\lambda} \dot{\mu}-4 \dot{\lambda}-3 \dot{\lambda}^{2}-2 \ddot{\lambda}-1, \\
0 & =2 \dot{\lambda} \dot{\mu}+\dot{\lambda}^{2} \\
0 & =\ddot{\lambda}+\dot{\lambda}^{2}+\dot{\lambda}-\dot{\mu}-\dot{\lambda} \dot{\mu}-\dot{\lambda} \dot{\nu} \\
e^{2 \mu} p & =1+2 \dot{\lambda}+\dot{\lambda}^{2}+2 \dot{\nu}+2 \dot{\lambda} \dot{\nu} \\
0 & =2 \dot{\lambda} \dot{\nu}-2 \ddot{\lambda}-3 \dot{\lambda}^{2}-2 \dot{\lambda}, \\
e^{2 \mu} p & =\ddot{\lambda}+\dot{\lambda}^{2}+\dot{\lambda}+\dot{\lambda} \dot{\nu}+\ddot{\nu}+\dot{\nu}^{2}-\dot{\mu}-\dot{\lambda} \dot{\mu}-\dot{\nu} \dot{\mu}, \\
0 & =-\ddot{\lambda}-\dot{\lambda}^{2}-\dot{\lambda}-\ddot{\mu}-\dot{\mu}^{2}-\dot{\mu}+\dot{\lambda} \dot{\nu}-\dot{\lambda} \dot{\mu}+\dot{\mu} \dot{\nu} .
\end{aligned}
$$


Here dot means derivative with respect to $\ln (\xi)$.

\subsubsection{Equation of State (3)}

If a perfect fluid satisfies $\operatorname{EOS}(3)$, Eqs.(20) and (21) yield that

$$
p=k \rho . \quad[\text { Case } I]
$$

From Eq.(25), we have two possibilities either $\dot{\lambda}=0$ or $\dot{\lambda}=-2 \dot{\mu}$. For the first possibility, we obtain the following vacuum solution

$$
\begin{aligned}
& \nu=\ln \left(c_{0} \xi^{(1 \mp \sqrt{2})}\right), \quad \mu=c_{1}, \quad \lambda=c_{2}, \\
& \rho=\text { constant }, \quad k=-3 \pm \sqrt{2} .
\end{aligned}
$$

The corresponding metric is

$$
d s^{2}=\left(\frac{x}{t}\right)^{(2 \mp 2 \sqrt{2})} d t^{2}-d x^{2}-x^{2}\left(d y^{2}+d z^{2}\right) .
$$

The second possibility leads to contradiction.

\subsection{Self-Similarity of the Second Kind}

It follows from the EFEs that the energy density $\rho$ and pressure $p$ can be written as

$$
\begin{aligned}
\kappa \rho & =\frac{1}{x^{2}}\left[\rho_{1}(\xi)+\frac{x^{2}}{t^{2}} \rho_{2}(\xi)\right], \\
\kappa p & =\frac{1}{x^{2}}\left[p_{1}(\xi)+\frac{x^{2}}{t^{2}} p_{2}(\xi)\right],
\end{aligned}
$$

where the self-similar variable is $\xi=x /(\alpha t)^{\frac{1}{\alpha}}$. When the EFEs and the equations of motion for the matter field are satisfied for $O\left[\left(\frac{x}{t}\right)^{0}\right]$ and $O\left[\left(\frac{x}{t}\right)^{2}\right]$ terms separately, we obtain the following ODEs

$$
\begin{aligned}
\dot{\rho_{1}} & =-(\dot{\mu}+2 \dot{\lambda})\left(\rho_{1}+p_{1}\right) \\
\dot{\rho_{2}}+2 \alpha \rho_{2} & =-(\dot{\mu}+2 \dot{\lambda})\left(\rho_{2}+p_{2}\right) \\
-\dot{p_{1}}+2 p_{1} & =\dot{\nu}\left(\rho_{1}+p_{1}\right) \\
-\dot{p_{2}} & =\dot{\nu}\left(\rho_{2}+p_{2}\right) \\
e^{2 \mu} \rho_{1} & =2 \dot{\mu}+2 \dot{\mu} \dot{\lambda}-4 \dot{\lambda}-3 \dot{\lambda}^{2}-2 \ddot{\lambda}-1
\end{aligned}
$$




$$
\begin{aligned}
\alpha^{2} e^{2 \nu} \rho_{2} & =2 \dot{\mu} \dot{\lambda}+\dot{\lambda}^{2}, \\
0 & =\ddot{\lambda}+\dot{\lambda}^{2}+\dot{\lambda}-\dot{\mu}-\dot{\lambda} \dot{\nu}-\dot{\lambda} \dot{\mu}, \\
e^{2 \mu} p_{1} & =1+2 \dot{\lambda}+\dot{\lambda}^{2}+2 \dot{\nu}+2 \dot{\lambda} \dot{\nu}, \\
\alpha^{2} e^{2 \nu} p_{2} & =-2 \ddot{\lambda}-3 \dot{\lambda}^{2}-2 \alpha \dot{\lambda}+2 \dot{\lambda} \dot{\nu}, \\
e^{2 \mu} p_{1} & =\ddot{\lambda}+\dot{\lambda}^{2}+\dot{\lambda}+\dot{\lambda} \dot{\nu}+\ddot{\nu}+\dot{\nu}^{2}-\dot{\mu}-\dot{\mu} \dot{\lambda}-\dot{\nu} \dot{\mu}, \\
\alpha^{2} e^{2 \nu} p_{2} & =-\ddot{\lambda}-\dot{\lambda}^{2}-\alpha \dot{\lambda}-\ddot{\mu}-\dot{\mu}^{2}-\alpha \dot{\mu}+\dot{\lambda} \dot{\nu}+\dot{\mu} \dot{\nu}-\dot{\lambda} \dot{\mu} .
\end{aligned}
$$

\subsubsection{Equations of State (1) and (2)}

When a perfect fluid satisfies $\operatorname{EOS}(1)$ for $k \neq 0$ and $\gamma \neq 0,1$, Eqs.(34) and (35) become

$$
\alpha=\gamma, \quad p_{1}=0=\rho_{2}, \quad p_{2}=\frac{k}{(8 \pi G)^{(\gamma-1)} \gamma^{2}} \xi^{-2 \gamma} \rho_{1}^{\gamma}, \quad[\text { Case } I]
$$

or

$$
\left.\alpha=\frac{1}{\gamma}, \quad p_{2}=0=\rho_{1}, \quad p_{1}=\frac{k}{(8 \pi G)^{(\gamma-1)} \gamma^{2 \gamma}} \xi^{2} \rho_{2}^{\gamma} . \quad \text { [Case } I I\right]
$$

For a perfect fluid with $\operatorname{EOS}(2)$ and $k \neq 0, \gamma \neq 0,1$, it follows from Eqs.(34) and (35) that

$$
\alpha=\gamma, \quad p_{1}=0, \quad p_{2}=\frac{k}{m_{b} \gamma(8 \pi G)^{(\gamma-1)} \gamma^{2}} \xi^{-2 \gamma} \rho_{1}^{\gamma}=(\gamma-1) \rho_{2}, \quad[\text { Case III }]
$$

or

$$
\alpha=\frac{1}{\gamma}, \quad p_{2}=0, \quad p_{1}=\frac{k}{m_{b}^{\gamma}(8 \pi G)^{(\gamma-1)} \gamma^{2 \gamma}} \xi^{2} \rho_{2}^{\gamma}=(\gamma-1) \rho_{1} . \quad[\text { Case } I V]
$$

In the Case $I$, Eq.(37) gives rise to two possibilities, i.e., either $\dot{\mu}=-2 \dot{\lambda}$ or $p_{2}=0$. For the first possibility we meet a contradiction. In the second option, we obtain the following vacuum solution

$$
\begin{gathered}
\nu=c_{1}, \quad \mu=\frac{1}{2} \ln \xi+c_{2}, \quad \lambda=-\ln \xi+c_{3}, \\
\rho_{1}=0=p_{1}, \quad \rho_{2}=0=p_{2}, \quad \alpha=\frac{3}{2} .
\end{gathered}
$$


The corresponding metric is

$$
d s^{2}=d t^{2}-\frac{2^{2 / 3} x}{(3 t)^{2 / 3}} d x^{2}-\left(\frac{3 t}{2}\right)^{4 / 3}\left(d y^{2}+d z^{2}\right) .
$$

For the Case $I I$, Eq.(36) shows that either $\dot{\mu}=-2 \dot{\lambda}$ or $p_{1}=0$. The first possibility leads to contradiction and the second possibility yields the same solution as given by Eq.(51).

In the Case $I I I$, Eq. (38) implies that either $\rho_{1}=0$ or $\dot{\nu}=0$. For the first option, Eq.(41) implies that either $\dot{\lambda}=0$ or $\dot{\lambda}=-2 \dot{\mu}$. The case when $\dot{\lambda}=0$ gives a contradiction and the option $\dot{\lambda}=-2 \dot{\mu}$ implies the same solution as given by Eq.(51). The second case $\dot{\nu}=0$ and the Case $I V$ also lead to the same solution as Eq.(51).

\subsubsection{Equation of State (3)}

For a perfect fluid satisfying EOS(3), Eqs.(34) and (35) yield that

$$
p_{1}=k \rho_{1}, \quad p_{2}=k \rho_{2} . \quad[\text { Case } V]
$$

This implies two options either $k=-1$ or $k \neq-1$. For $k=-1$, Eqs.(36)-(46) lead to the same solution as for $\operatorname{EOS}(1)$ and $\operatorname{EOS}(2)$ given by Eq.(51). For $k \neq-1$, the case $\rho_{1} \neq 0, \rho_{2} \neq 0$ leads to a contradiction. The case, when $\rho_{1}=0$ and $\rho_{2}$ is arbitrary, implies that

$$
\begin{gathered}
\nu=c_{1}, \quad \mu=\frac{3}{2} \ln \xi+c_{2}, \quad \lambda=-\ln \xi+c_{3}, \\
\rho_{1}=0=p_{1}, \quad \rho_{2}=\text { constant }=p_{2}, \quad \alpha=\frac{1}{2} .
\end{gathered}
$$

The resulting plane symmetric metric becomes

$$
d s^{2}=d t^{2}-\frac{64 x^{3}}{t^{6}} d x^{2}-\frac{t^{4}}{16}\left(d y^{2}+d z^{2}\right) .
$$

For the case when $\rho_{2}=0$ and $\rho_{1}$ is arbitrary, Eq.(41) implies that either $\dot{\lambda}=0$ or $\dot{\lambda}=-2 \dot{\mu}$. For the first possibility, it follows that

$$
\begin{aligned}
\nu & =\frac{2 k}{k+1} \ln \xi+c_{1}, \quad \mu=c_{2}, \quad \lambda=c_{3}, \quad p_{1}=\text { constant } \\
\rho_{2} & =0=p_{2}, \quad k=-3 \pm 2 \sqrt{2}
\end{aligned}
$$


and hence the plane symmetric spacetime will take the following form

$$
d s^{2}=\left(\frac{x}{(\alpha t)^{1 / \alpha}}\right)^{\frac{4 k}{k+1}} d t^{2}-d x^{2}-x^{2}\left(d y^{2}+d z^{2}\right) .
$$

For the second possibility, Eqs.(42) and (44) further imply two possibilities either $\dot{\mu}=0$ or $\alpha=\frac{3}{2}$. When $\dot{\mu}=0$ we obtain the same solution as Eq.(56). For $\alpha=\frac{3}{2}$, we can solve the system of equations by assuming either $\dot{\mu}=0$ or $\dot{\nu}=0$. Assuming $\dot{\mu}=0$, we obtain the same solution as given by Eq.(56). If we take $\dot{\nu}=0$, we have a contradiction.

\subsection{Self-Similarity of the Zeroth Kind}

For this case, the EFEs show that the quantities $\rho$ and $p$ must be of the form

$$
\begin{aligned}
\kappa \rho & =\frac{1}{x^{2}}\left[\rho_{1}(\xi)+x^{2} \rho_{2}(\xi)\right], \\
\kappa p & =\frac{1}{x^{2}}\left[p_{1}(\xi)+x^{2} p_{2}(\xi)\right],
\end{aligned}
$$

where the self-similar variable is $\xi=\frac{x}{e^{t}}$. Assuming that the EFEs and the equations of motion for the matter field are satisfied for $O\left[(x)^{0}\right]$ and $O\left[(x)^{2}\right]$ terms separately, it follows that

$$
\begin{aligned}
\dot{\rho_{1}} & =-(\dot{\mu}+2 \dot{\lambda})\left(\rho_{1}+p_{1}\right), \\
\dot{\rho_{2}} & =-(\dot{\mu}+2 \dot{\lambda})\left(\rho_{2}+p_{2}\right), \\
-\dot{p_{1}}+2 p_{1} & =\dot{\nu}\left(\rho_{1}+p_{1}\right), \\
-\dot{p_{2}} & =\dot{\nu}\left(\rho_{2}+p_{2}\right), \\
e^{2 \mu} \rho_{1} & =2 \dot{\mu}-4 \dot{\lambda}-3 \dot{\lambda}^{2}-2 \ddot{\lambda}+2 \dot{\lambda} \dot{\mu}-1, \\
e^{2 \nu} \rho_{2} & =2 \dot{\lambda} \dot{\mu}+\dot{\lambda}^{2} \\
0 & =\ddot{\lambda}+\dot{\lambda}^{2}+\dot{\lambda}-\dot{\mu}-\dot{\lambda} \dot{\mu}-\dot{\lambda} \dot{\nu}, \\
e^{2 \mu} p_{1} & =1+2 \dot{\lambda}+\dot{\lambda}^{2}+2 \dot{\nu}+2 \dot{\lambda} \dot{\nu}, \\
e^{2 \nu} p_{2} & =2 \dot{\lambda} \dot{\nu}-2 \ddot{\lambda}-3 \dot{\lambda}^{2}, \\
e^{2 \mu} p_{1} & =\ddot{\lambda}+\dot{\lambda}^{2}+\dot{\lambda}+\dot{\lambda} \dot{\nu}+\ddot{\nu}+\dot{\nu}^{2}-\dot{\mu}-\dot{\lambda} \dot{\mu}-\dot{\mu} \dot{\nu}, \\
e^{2 \nu} p_{2} & =-\ddot{\lambda}-\dot{\lambda}^{2}+\dot{\lambda} \dot{\nu}-\dot{\lambda} \dot{\mu}+\dot{\mu} \dot{\nu}-\dot{\mu}^{2}-\ddot{\mu} .
\end{aligned}
$$




\subsection{1 $\operatorname{EOS}(1)$ and $\operatorname{EOS}(2)$}

Here Eqs.(58) and (59) imply that

$$
p_{1}=0=\rho_{1}, \quad p_{2}=\frac{k}{(8 \pi G)^{(\gamma-1)}} \rho_{2}^{\gamma}, \quad[\text { Case } I]
$$

For $\operatorname{EOS}(2)$, it turns out that

$$
p_{1}=0=\rho_{1}, \quad p_{2}=\frac{k}{m_{b}^{\gamma}(8 \pi G)^{(\gamma-1)}}\left[\rho_{2}-\frac{p_{2}}{(\gamma-1)}\right]^{\gamma} . \quad[\text { Case II }]
$$

In both cases, we get the same set of equations which on solving yield the following solution for both $\operatorname{EOS}(1)$ and $\operatorname{EOS}(2)$

$$
\begin{aligned}
\nu & =c_{1}, \quad \mu=-\ln \xi+\ln \left(\xi^{3}-c_{3}\right)+c_{2}, \quad \lambda=-\ln \xi+c_{4}, \\
\rho_{1} & =0=p_{1}, \quad \rho_{2}=-\frac{3\left(\xi^{3}+c_{3}\right)}{e^{2 c_{1}}\left(\xi^{3}-c_{3}\right)}, \quad p_{2}=\text { constant } .
\end{aligned}
$$

The corresponding metric is

$$
d s^{2}=d t^{2}-\left(\frac{x^{3}-c_{3} e^{3 t}}{x e^{2 t}}\right)^{2} d x^{2}-e^{2 t}\left(d y^{2}+d z^{2}\right) .
$$

\subsection{2 $\operatorname{EOS}(3)$}

Here it follows from Eqs.(58) and (59) that

$$
p_{1}=k \rho_{1}, \quad p_{2}=k \rho_{2} .
$$

Proceeding in a similar fashion as in the case of self-similarity of the second kind with $\operatorname{EOS}(3)$, we obtain, for $k=-1$, the following solution

$$
\begin{aligned}
\nu & =c_{1}, \quad \mu=-\ln \xi+c_{2}, \quad \lambda=-\ln \xi+c_{3}, \\
\rho_{1} & =0=p_{1}, \quad \rho_{2}=\text { constant }=-p_{2} .
\end{aligned}
$$

The corresponding plane symmetric metric is

$$
d s^{2}=d t^{2}-\frac{e^{2 t}}{x^{2}} d x^{2}-e^{2 t}\left(d y^{2}+d z^{2}\right)
$$


The case $k \neq-1$ again leads to three options either $\rho_{1} \neq 0 \neq \rho_{2}, \rho_{1}=0$ or $\rho_{2}=0$. The first case gives a contradiction. For the second option, we obtain the following solution

$$
\begin{aligned}
\nu & =c_{1}, \quad \mu=2 \ln \xi+c_{2}, \quad \lambda=-\ln \xi+c_{3}, \\
\rho_{1} & =0=p_{1}, \quad \rho_{2}=\text { constant }=p_{2} .
\end{aligned}
$$

The plane symmetric metric for this solution becomes

$$
d s^{2}=d t^{2}-x^{4} e^{-4 t} d x^{2}-e^{2 t}\left(d y^{2}+d z^{2}\right) .
$$

The case, when $\rho_{2}=0$, Eq.(65) yields two possibilities either $\dot{\lambda}=0$ or $\dot{\lambda}=-2 \dot{\mu}$. For both possibilities, we obtain the same solution as in the case of the second kind with $\operatorname{EOS}(3)$ given by Eq.(56) $(\alpha=0)$. The corresponding metric is

$$
d s^{2}=\left(x e^{-t}\right)^{\frac{4 k}{k+1}} d t^{2}-d x^{2}-e^{2 t}\left(d y^{2}+d z^{2}\right) .
$$

\subsection{Self-Similarity of the Infinite Kind}

In this case, the EFEs indicate that the quantities $\rho$ and $p$ must be of the following form

$$
\begin{aligned}
\kappa \rho & =\rho_{1}(\xi)+\frac{1}{t^{2}} \rho_{2}(\xi), \\
\kappa p & =p_{1}(\xi)+\frac{1}{t^{2}} p_{2}(\xi),
\end{aligned}
$$

where $\xi=\frac{x}{t}$. The requirement that the EFEs and the equations of motion for the matter field are satisfied for $O\left[(t)^{0}\right]$ and $O\left[(t)^{-2}\right]$ terms separately leads to the following equations

$$
\begin{aligned}
\dot{\rho_{1}} & =-(\dot{\mu}+2 \dot{\lambda})\left(\rho_{1}+p_{1}\right), \\
\rho_{2}+2 \rho_{2} & =-(\dot{\mu}+2 \dot{\lambda})\left(\rho_{2}+p_{2}\right), \\
-\dot{p_{1}} & =\dot{\nu}\left(\rho_{1}+p_{1}\right), \\
-\dot{p_{2}} & =\dot{\nu}\left(\rho_{2}+p_{2}\right), \\
e^{2 \mu} \rho_{1} & =2 \dot{\lambda} \dot{\mu}-3 \dot{\lambda}^{2}-2 \ddot{\lambda}, \\
e^{2 \nu} \rho_{2} & =2 \dot{\lambda} \dot{\mu}+\dot{\lambda}^{2}, \\
0 & =\ddot{\lambda}+\dot{\lambda}^{2}-\dot{\lambda} \dot{\nu}-\dot{\lambda} \dot{\mu},
\end{aligned}
$$




$$
\begin{aligned}
e^{2 \mu} p_{1} & =\dot{\lambda}^{2}+2 \dot{\lambda} \dot{\nu}, \\
e^{2 \nu} p_{2} & =-2 \ddot{\lambda}-3 \dot{\lambda}^{2}-2 \dot{\lambda}+2 \dot{\lambda} \dot{\nu}, \\
e^{2 \mu} p_{1} & =\ddot{\lambda}+\dot{\lambda}^{2}+\dot{\lambda} \dot{\nu}+\ddot{\nu}+\dot{\nu}^{2}+\dot{\lambda} \dot{\mu}-\dot{\mu} \dot{\nu}, \\
e^{2 \nu} p_{2} & =-\ddot{\lambda}-\dot{\lambda}^{2}-\dot{\lambda}+\dot{\lambda} \dot{\nu}-\ddot{\mu}-\dot{\mu}^{2}-\dot{\mu}+\dot{\mu} \dot{\nu}-\dot{\lambda} \dot{\mu} .
\end{aligned}
$$

\subsection{1 $\quad \operatorname{EOS}(1)$ and $\operatorname{EOS}(2)$}

For EOS(1), Eqs.(81) and (82) imply that

$$
p_{2}=0=\rho_{2}, \quad p_{1}=k(8 \pi G)^{(1-\gamma)} \rho_{1}^{\gamma} . \quad[\text { Case } I]
$$

$\operatorname{EOS}(2)$ implies that

$$
p_{2}=0=\rho_{2}, \quad p_{1}=\frac{k}{m_{b}^{\gamma}(8 \pi G)^{(\gamma-1)}}\left(\rho_{1}-\frac{p_{1}}{(\gamma-1)}\right)^{\gamma} . \quad[\text { Case } I I]
$$

In both cases, Eq.(88) shows that either $\lambda=$ constant or $\dot{\lambda}=-2 \dot{\mu}$. If $\lambda=$ constant, Eqs.(86) and (90), respectively, imply that $\rho_{1}=0=p_{1}$ and we are left with Eqs.(92) and (93). Solving these two equations lead to $\ddot{\nu}+\dot{\nu}^{2}-\ddot{\mu}-\dot{\mu}^{2}-\dot{\mu}=0$ which satisfies for four different possibilities. For $\dot{\mu}=0=\dot{\nu}$, we trivially get Minkowski spacetime. For $\dot{\mu}=0$, the solution turns out to be

$$
\begin{aligned}
\nu & =\ln \left(\ln \xi-\ln c_{1}\right)+c_{2}, \quad \mu=c_{3}, \quad \lambda=c_{4}, \\
\rho_{1} & =0=p_{1}, \quad \rho_{2}=0=p_{2} .
\end{aligned}
$$

The metric will be

$$
d s^{2}=\left[\ln \left(\frac{x}{c_{1} t}\right)\right]^{2} d t^{2}-\frac{1}{x^{2}} d x^{2}-\left(d y^{2}+d z^{2}\right), \quad\left(c_{1} \neq 0\right) .
$$

In the case $\dot{\nu}=0$, we obtain

$$
\begin{aligned}
\nu & =c_{1}, \quad \mu=\ln \left(\ln \xi-\ln c_{2}\right)+c_{3}, \quad \lambda=c_{4}, \\
\rho_{1} & =0=p_{1}, \quad \rho_{2}=0=p_{2} .
\end{aligned}
$$

The corresponding metric is

$$
d s^{2}=d t^{2}-\frac{1}{x^{2}}\left[\ln \left(\frac{x}{c_{2} t}\right)\right]^{2} d x^{2}-\left(d y^{2}+d z^{2}\right), \quad\left(c_{2} \neq 0\right) .
$$

Finally, for the last possibility $\ddot{\nu}+\dot{\nu}^{2}=0$ and $-\ddot{\mu}-\dot{\mu}^{2}-\dot{\mu}=0$, Eqs.(92) and (93) imply $\dot{\mu} \dot{\nu}=0$ and again we have the above possibilities. The second case, when $\dot{\lambda}=-2 \dot{\mu}$, gives the same solution as given by Eq.(97). 


\subsection{2 $\operatorname{EOS}(3)$}

Eqs.(81) and (82) imply that

$$
p_{1}=k \rho_{1}, \quad p_{2}=k \rho_{2} . \quad[\text { Case } I I I]
$$

When $k=-1$, this gives rise to the same solution as for $\operatorname{EOS}(1)$ and $\operatorname{EOS}(2)$. The second case, i.e., $k \neq-1$ also leads to the same results as in $\operatorname{EOS}(1)$ and $\operatorname{EOS}(2)$.

\section{Tilted Dust Case}

\subsection{Self-Similarity of the First Kind}

When we take $p=0$ in Eqs.(22)-(30) for the tilted perfect fluid case with self-similarity of the first kind, Eq.(23) gives either $\dot{\nu}=0$ or $\rho=0$. Both the cases yield contradiction.

\subsection{Self-Similarity of the Second Kind}

Here for $p_{1}=0=p_{2}$, Eqs.(38) and (39) imply that either $\nu=$ constant or $\rho_{1}=0=\rho_{2}$. For the first possibility, we obtain the following solution

$$
\begin{aligned}
\nu & =c_{1}, \quad \mu=\ln \left(c_{3} \xi^{-1 / 2}\left(\xi^{3 / 2} \mp 2 c_{2}^{3 / 2}\right)\right), \quad \lambda=-\ln \xi+c_{4}, \\
\rho_{1} & =0=p_{1}, \quad \rho_{2}=\frac{2}{3 c_{5}}\left(2-3\left(\frac{\xi^{3 / 2}}{\xi^{3 / 2} \mp 2 c_{2}{ }^{3 / 2}}\right)\right), \quad p_{2}=0, \\
\alpha & =\frac{3}{2} .
\end{aligned}
$$

The corresponding metric is

$$
d s^{2}=d t^{2}-\left(\frac{3 t^{2 / 3}}{2^{2 / 3} x}\right)\left(\frac{2 x^{3 / 2}}{3 t} \mp 2 c_{2}{ }^{3 / 2}\right)^{2} d x^{2}-\left(\frac{3 t}{2}\right)^{4 / 3}\left(d y^{2}+d z^{2}\right) .
$$

The second possibility leads to the same solution as given by Eq.(51).

\subsection{Self-Similarity of the Zeroth Kind}

This case gives contradiction and hence there is no solution. 


\subsection{Self-Similarity of the Infinite Kind}

In this case, Eqs.(85) and (86) imply that either $\nu=$ constant or $\rho_{1}=0=\rho_{2}$.

In the first case, we obtain the following solution

$$
\begin{aligned}
\nu & =c_{1}, \quad \mu=-\ln \xi+\ln \left(\xi-c_{2}\right)+c_{3}, \quad \lambda=c_{4}, \\
\rho_{1} & =0=\rho_{2} .
\end{aligned}
$$

The corresponding metric is

$$
d s^{2}=d t^{2}-\frac{\left(x-c_{2} t\right)^{2}}{x^{4}} d x^{2}-\left(d y^{2}+d z^{2}\right) .
$$

For the second case, when $\rho_{1}=0=\rho_{2}$, Eqs.(88) and (90) imply that either $\dot{\lambda}=0$ or $\dot{\mu}=\dot{\nu}, \dot{\lambda}=-2 \dot{\mu}$. The first option yields exactly the same result as for the tilted perfect fluid with self-similarity of the infinite kind using EOS(1) and $\operatorname{EOS}(2)$ and are given by Eqs.(96), (98) and Minkowski spacetime. The other possibility implies a Minkowski spacetime.

\section{Orthogonal Perfect Fluid and Dust Cases}

Here the self-similar variable is $\xi=t$ in each kind. The EFEs and the equations of motion for the perfect fluid of the first kind gives the following set of equations

$$
\begin{aligned}
\dot{\mu} & =0, \\
e^{2 \nu}\left(e^{-2 \mu}+\rho\right) & =\lambda^{\prime 2} \\
e^{2 \nu}\left(3 e^{-2 \mu}-p\right) & =3 \lambda^{\prime 2}+2 \lambda^{\prime \prime}-2 \lambda^{\prime} \nu^{\prime} \\
e^{2 \nu}\left(e^{-2 \mu}-p\right) & =\lambda^{\prime \prime}+\lambda^{\prime 2}-\lambda^{\prime} \nu^{\prime} \\
2 \lambda^{\prime}(\rho+p) & =-\rho_{1}^{\prime} \\
\rho & =p
\end{aligned}
$$

where prime indicates derivative with respect to $\xi=t$. Eq.(110) gives an equation of state for this system of equations. Solving these equations simultaneously, we arrive at the following solution

$$
\begin{aligned}
\nu & =\ln \left(\frac{p^{\prime}}{4 p \sqrt{\left(c_{0}+p\right)}}\right), \quad \mu=c_{1}, \quad \lambda=-\frac{1}{4} \ln (p)+\ln \left(c_{2}\right), \\
\rho & =p, \quad p^{\prime 2} p-2(1+p)\left(p^{\prime \prime} p-p^{\prime 2}\right)=0 .
\end{aligned}
$$


For the perfect fluid case of the second and zeroth kinds, we obtain contradiction. The perfect fluid case of the infinite kind gives Minkowski spacetime.

For the dust case, we take $p=0$ in the equations for the perfect fluid case. In the self-similarity of the first kind, Eq.(110) shows that the resulting spacetime must be vacuum. Eq.(106) gives $e^{2 \nu} e^{2 \mu}=\lambda^{\prime 2}$ and we obtain

$$
\nu=\nu(\xi), \quad \lambda=c_{0} \int e^{\nu(\xi)} d \xi, \quad \mu=c_{1}, \quad \rho=0=p .
$$

The metric becomes

$$
d s^{2}=x^{2} e^{2 \nu(t)} d t^{2}-d x^{2}-x^{2} e^{2 c_{0} \int e^{\nu(t)} d t}\left(d y^{2}+d z^{2}\right) .
$$

For the self-similarity of the second, zeroth and infinite kinds, we arrive at a contradiction due to one or the other reason and hence there is no solution.

\section{Parallel Perfect Fluid Case}

\subsection{Self-Similarity of the First Kind}

Here the self-similar variable is $\xi=x$ and the metric functions are given by Eq.(17). A set of ODEs in terms of $\xi$ are

$$
\begin{aligned}
\nu^{\prime} & =0 \\
\rho & =3 e^{-2 \nu}+e^{-2 \mu}\left(2 \lambda^{\prime} \mu^{\prime}-3 \lambda^{2}-2 \lambda^{\prime \prime}\right) \\
p & =e^{-2 \mu}\left(\lambda^{\prime 2}+2 \lambda^{\prime} \nu^{\prime}\right)-e^{-2 \nu} \\
p & =e^{-2 \mu}\left(\lambda^{\prime \prime}+\lambda^{\prime 2}+\lambda^{\prime} \nu^{\prime}+\nu^{\prime \prime}+\nu^{2}-\lambda^{\prime} \mu^{\prime}-\mu^{\prime} \nu^{\prime}\right)-e^{-2 \nu} \\
0 & =\rho+3 p \\
-p^{\prime} & =\nu^{\prime}(\rho+p)
\end{aligned}
$$

Here prime denotes derivative with respect to $\xi=x$. Eq.(118) indicates an equation of state. Using Eq.(114) in rest of the equations, we get $p^{\prime}=0$. Solving the remaining equations, we obtain

$$
\nu=c_{1}, \quad \mu=c_{2}, \quad \lambda=c_{3} \xi+c_{4}, \quad \rho=0=p
$$

and the corresponding spacetime is

$$
d s^{2}=d t^{2}-t^{2} d x^{2}-t^{2} e^{2 c_{3} x}\left(d y^{2}+d z^{2}\right) .
$$




\subsection{Self-Similarity of the Second Kind}

For this kind, the self-similar variable is also $\xi=x$ and the metric functions are given by Eq.(17). The EFEs imply that the quantities $\rho$ and $p$ must be of the form

$$
\begin{aligned}
& \kappa \rho=t^{-2} \rho_{1}(\xi)+t^{-2 \alpha} \rho_{2}(\xi), \\
& \kappa p=t^{-2} p_{1}(\xi)+t^{-2 \alpha} p_{2}(\xi) .
\end{aligned}
$$

A set of ODEs in terms of $\xi$ will be

$$
\begin{aligned}
\nu^{\prime} & =0, \\
e^{2 \mu} \rho_{1} & =2 \lambda^{\prime} \mu^{\prime}-3 \lambda^{\prime 2}-2 \lambda^{\prime \prime}, \\
\rho_{2} & =3 e^{-2 \nu}, \\
e^{2 \mu} p_{1} & =\lambda^{\prime 2}, \\
e^{2 \nu} p_{2} & =2 \alpha-3, \\
e^{2 \mu} p_{1} & =\lambda^{\prime \prime}+\lambda^{\prime 2}-\lambda^{\prime} \mu^{\prime}, \\
e^{2 \nu} p_{2} & =2 \alpha-3, \\
0 & =\rho_{1}+3 p_{1}, \\
0 & =(3-2 \alpha) \rho_{2}+3 p_{2}, \\
-p_{1}^{\prime} & =0, \\
-p_{2}^{\prime} & =0 .
\end{aligned}
$$

\subsection{1 $\operatorname{EOS}(1)$ and $\operatorname{EOS}(2)$}

When a perfect fluid satisfies EOS(1), Eqs.(122) and (123) imply that

$$
p_{2}=0=\rho_{1}, \quad \alpha=\frac{1}{\gamma}, \quad p_{1}=k(8 \pi G)^{(1-\gamma)} \rho_{2}{ }^{\gamma} . \quad[\text { Case } I]
$$

For $\operatorname{EOS}(2)$, it turns out that

$$
p_{2}=0, \quad \alpha=\frac{1}{\gamma}, \quad p_{1}=\frac{k}{m_{b}^{\gamma}(8 \pi G)^{(\gamma-1)}} \rho_{2}^{\gamma}=(\gamma-1) \rho_{1} . \quad[\text { Case } I I]
$$

The Case $I$ gives a contradiction and the Case $I I$ yields the following solution

$$
\begin{aligned}
\nu & =c_{1}, \quad \mu=\ln \lambda^{\prime}+c_{2}, \quad \lambda=\lambda(\xi) \\
\rho_{1} & =-3 p_{1}=\text { constant }, \quad \rho_{2}=\frac{3}{c_{0}^{2}}, \quad p_{2}=0, \quad \alpha=\frac{3}{2} .
\end{aligned}
$$


The spacetime becomes

$$
d s^{2}=t d t^{2}-t^{2} \lambda^{\prime 2} d x^{2}-t^{2} e^{2 \lambda(x)}\left(d y^{2}+d z^{2}\right) .
$$

\subsection{2 $\operatorname{EOS}(3)$}

For EOS(3), Eqs.(122) and (123) show that

$$
p_{1}=k \rho_{1}, \quad p_{2}=k \rho_{2} .
$$

Here Eqs.(131) and Eq.(132) imply that $\rho_{1}=0$ and Eq.(124) gives $\dot{\nu}=0$ while Eq.(127) implies that $\dot{\lambda}=0$. Solving the remaining equations, it turns out that

$$
\begin{aligned}
\nu & =c_{1}, \quad \mu=\mu(\xi), \quad \lambda=c_{2}, \quad \rho_{1}=0=p_{1}, \\
\rho_{2} & =\frac{3}{c_{0}}, \quad p_{2}=-\frac{(3-2 \alpha)}{c_{0}}, \quad k=-\frac{(3-2 \alpha)}{3} .
\end{aligned}
$$

This gives the following spacetime

$$
d s^{2}=t^{2(\alpha-1)} d t^{2}-t^{2} e^{2 \mu(x)} d x^{2}-t^{2}\left(d y^{2}+d z^{2}\right) .
$$

\subsection{Self-Similarity of the Zeroth Kind}

For this kind, the self-similar variable is again $\xi=x$ and the plane symmetric

metric functions are given by Eq.(17). The EFEs imply that the quantities $\rho$ and $p$ must be of the form

$$
\begin{aligned}
\kappa \rho & =t^{-2} \rho_{1}(\xi)+\rho_{2}(\xi) \\
\kappa p & =t^{-2} p_{1}(\xi)+p_{2}(\xi) .
\end{aligned}
$$

ODEs are

$$
\begin{aligned}
\nu^{\prime} & =0, \\
e^{2 \mu} \rho_{1} & =2 \lambda^{\prime} \mu^{\prime}-3 \lambda^{\prime 2}-2 \lambda^{\prime \prime}, \\
\rho_{2} & =3 e^{-2 \nu}, \\
e^{2 \mu} p_{1} & =\lambda^{\prime 2}, \\
e^{2 \nu} p_{2} & =-3, \\
e^{2 \mu} p_{1} & =\lambda^{\prime \prime}+\lambda^{\prime 2}-\lambda^{\prime} \mu^{\prime},
\end{aligned}
$$




$$
\begin{aligned}
e^{2 \nu} p_{2} & =-3, \\
0 & =\rho_{1}+3 p_{1}, \\
0 & =\rho_{2}+p_{2}, \\
-p_{1}^{\prime} & =0 \\
-p_{2}^{\prime} & =0 .
\end{aligned}
$$

\subsection{1 $\quad \operatorname{EOS}(1)$ and $\operatorname{EOS}(2)$}

In the case of $\operatorname{EOS(1),~Eqs.(142)~and~(143)~yield~}$

$$
\rho_{1}=0=p_{1}, \quad p_{2}=k(8 \pi G)^{(1-\gamma)} \rho_{2}^{\gamma} . \quad[\text { Case I }]
$$

For $\operatorname{EOS}(2)$, it turns out that

$$
p_{1}=0=\rho_{1}, \quad p_{2}=\frac{k}{m_{b}^{\gamma}(8 \pi G)^{(\gamma-1)}}\left[\rho_{2}-\frac{p_{2}}{(\gamma-1)}\right]^{\gamma}, \quad[\text { Case II }]
$$

In both the cases, we obtain the same solution as

$$
\begin{aligned}
\nu & =c_{1}, \quad \mu=\mu(\xi), \quad \lambda=c_{2}, \\
\rho_{1} & =0=p_{1}, \quad \rho_{2}=-p_{2}=\text { constant }
\end{aligned}
$$

and the resulting metric is

$$
d s^{2}=\frac{1}{t^{2}} d t^{2}-t^{2} e^{2 \mu(x)} d x^{2}-t^{2}\left(d y^{2}+d z^{2}\right) .
$$

\subsection{2 $\operatorname{EOS}(3)$}

Eqs.(142) and (143) show that

$$
p_{1}=k \rho_{1}, \quad p_{2}=k \rho_{2} .
$$

Here Eqs.(151) and (152) imply that either $\rho_{1}=0$ or $\rho_{2}=0$. Eq.(146) gives a contradiction for $\rho_{2}=0$ and hence $\rho_{1}=0$. Also, Eq.(151) shows that $k=-1$ hence this gives the same solution as in $\operatorname{EOS}(1)$ and $\operatorname{EOS}(2)$ given by Eq.(158). 


\subsection{Self-Similarity of the Infinite Kind}

Again we have the self-similar variable $\xi=x$ and the spacetime metric coefficients are given by Eq.(17). A set of ODEs will be

$$
\begin{aligned}
-e^{2 \mu} \rho & =3 \lambda^{2}+2 \lambda^{\prime \prime}-2 \lambda^{\prime} \mu^{\prime}, \\
e^{2 \mu} p & =\lambda^{\prime 2}+2 \lambda^{\prime} \nu^{\prime}, \\
e^{2 \mu} p & =\lambda^{\prime \prime}+\lambda^{\prime 2}+\lambda^{\prime} \nu^{\prime}+\nu^{\prime \prime}+\nu^{\prime 2}-\lambda^{\prime} \mu^{\prime}-\nu^{\prime} \mu^{\prime}, \\
-p^{\prime} & =\nu^{\prime}(\rho+p) .
\end{aligned}
$$

We consider the following four possibilities to solve the above set of equations.

$$
\begin{array}{rll}
(i) & \nu^{\prime}=\mu^{\prime}, & \text { (ii) } \quad \nu^{\prime}=\lambda^{\prime}, \\
(\text { iii }) & \lambda^{\prime}=\mu^{\prime}, & \text { (iv) } \quad \nu^{\prime}=\lambda^{\prime}=\mu^{\prime} .
\end{array}
$$

The first case gives the following solution

$$
\begin{aligned}
\nu & =\mu=c_{1}, \quad \lambda=c_{2} \xi+c_{3}, \\
\rho & =-3 p=\text { constant }
\end{aligned}
$$

and the corresponding metric is

$$
d s^{2}=d t^{2}-d x^{2}-e^{2 x}\left(d y^{2}+d z^{2}\right)
$$

The second case corresponds to Minkowski spacetime. For the case (iii), we obtain the following solution

$$
\begin{aligned}
\nu & =c_{1}, \quad \lambda=\mu=c_{3}-\ln \left(\xi-c_{2}\right), \\
\rho & =3 p, \quad p=-1
\end{aligned}
$$

and the metric is given by

$$
d s^{2}=d t^{2}-\frac{1}{x^{2}}\left(d x^{2}+d y^{2}+d z^{2}\right) .
$$

The last case yields Minkowski spacetime.

\section{$7 \quad$ Parallel Dust case}

\subsection{Self-Similarity of the First Kind}

Setting $p=0$ in the equations for the parallel perfect fluid case with selfsimilarity of the first kind, we finally have a contradiction and hence we do not have any self-similar solution. 


\subsection{Self-Similarity of the Second Kind}

For $p_{1}=0=p_{2}$, Eqs.(124) and (134) show that $\nu=$ constant $=\lambda$ respectively and we get the same solution as given by Eq.(157) with $\rho_{2}=0=p_{2}$ and $\alpha=\frac{3}{2}$ but the corresponding metric is

$$
d s^{2}=t d t^{2}-t^{2} e^{2 \mu(x)} d x^{2}-t^{2}\left(d y^{2}+d z^{2}\right) .
$$

\subsection{Self-Similarity of the Zeroth Kind}

When we take $p_{1}=0=p_{2}$, Eqs.(148) and (150) lead to contradiction.

\subsection{Self-Similarity of the Infinite Kind}

For $p=0$, Eq.(163) shows that either $\nu=$ constant or $\rho=0$. In the first case, the resulting spacetime is Minkowski. For $\rho=0$, Eq.(161) implies that either $\lambda^{\prime}=0$ or $\lambda^{\prime}=-2 \nu^{\prime}$. When $\lambda^{\prime}=0$, we obtain $\nu^{\prime \prime}+\nu^{\prime 2}-\mu^{\prime} \nu^{\prime}=0$ which implies that either $\nu^{\prime}=0$ or $\mu^{\prime}=0$. For the first possibility, we obtain Minkowski spacetime. For the second possibility, we get the following vacuum solution

$$
\nu=\ln \left(c_{2}\left(\xi-c_{1}\right)\right), \quad \mu=c_{3}, \quad \lambda=c_{4}, \quad \rho=0=p .
$$

The metric for this spacetime is

$$
d s^{2}=\left(c_{2}\left(x-c_{1}\right)\right)^{2} d t^{2}-\left(d x^{2}+d y^{2}+d z^{2}\right) .
$$

For $\lambda^{\prime}=-2 \nu^{\prime}$, Eqs.(160) and (162) imply that $2 \nu^{\prime \prime}-3 \nu^{\prime 2}-\mu^{\prime} \nu^{\prime}=0$ which gives either $\nu^{\prime}=0$ or $\mu^{\prime}=0$. The first possibility leads to the Minkowski spacetime and the second possibility gives the following vacuum solution

$$
\nu=\ln \left(\frac{c_{2}}{\left(3 x-c_{1}\right)^{1 / 3}}\right), \quad \mu=c_{3}, \quad \lambda=c_{4}, \quad \rho=0=p
$$

and the corresponding metric is

$$
d s^{2}=\left(\frac{c_{2}{ }^{2}}{\left(3 x-c_{1}\right)^{2 / 3}}\right) d t^{2}-\left(d x^{2}+d y^{2}+d z^{2}\right)
$$




\section{Summary and Discussion}

Recent literature $[3-5,7,17-20]$ indicates keen interest in the self-similar solutions and their physical features. Maeda et al. [3-5] have classified the spherically symmetric KSS perfect fluid and dust solutions. Sharif and Sehar [7] have extended this analysis for the classification of the KSS cylindrically symmetric solutions. Recently, the same authors [21] have explored the KSS solutions for the plane symmetric spacetimes under certain restriction, i.e., $\mu=0$ for the sake of simplicity. Consequently, the classification was incomplete in the sense that we were missing many such cases where solution can be possible. This paper deals with the most general plane symmetric spacetimes and provides self-similar solutions even in those cases where we obtain null results [21]. We have classified KSS perfect fluid and dust solutions for the cases when KSS vector is tilted, orthogonal and parallel to the fluid flow by using $\operatorname{EOS}(1), \operatorname{EOS}(2)$ and $\operatorname{EOS}(3)$. This gives rise to twenty four plane symmetric self-similar solutions out of which we obtain sixteen independent solutions.

It is found that $\operatorname{EOS}(1)$ and $\operatorname{EOS}(2)$ are incompatible with the selfsimilarity of the first kind in the tilted perfect fluid case. For $\operatorname{EOS}(3)$, we obtain solution with constant density. For the self-similarity of the second kind with $\operatorname{EOS}(1)$ and $\operatorname{EOS}(2)$, we obtain a vacuum solution. For $\operatorname{EOS}(3)$ with $k=-1$, it follows the same solution as for $\operatorname{EOS}(1)$ and $\operatorname{EOS}(2)$. The case $k \neq-1$ leads to two self-similar solutions one of these $\left(\rho_{1}=0\right)$ represents a stiff fluid. The zeroth kind with $\operatorname{EOS}(1)$ and $\operatorname{EOS}(2)$ yields a solution. $\operatorname{EOS}(3)$ gives three solution, one is vacuum solution and other is a stiff fluid solution. In the case of the infinite kind for $\operatorname{EOS}(1)$ and $\operatorname{EOS}(2)$, we find three vacuum solutions while $\operatorname{EOS}(3)$ also leads to vacuum solutions both for $k=-1$ and $k \neq-1$.

For the tilted dust case with self-similarity of the second kind, we obtain the same solution as for the tilted perfect fluid with $\operatorname{EOS}(1)$ and $\operatorname{EOS}(2)$ and a dust solution for $\alpha=3 / 2$. The self-similarity of the infinite kind leads to four vacuum solutions one of them is Minkowski spacetime. There is no solution in any other kind.

In the orthogonal perfect fluid with self-similarity of the first kind we obtain a solution in terms of pressure and with self-similarity of the infinite kind we obtain Minkowski spacetime. Any other kind does not provide any solution. The orthogonal dust case with self-similarity of the first kind yields a vacuum spacetime given by Eq.(112). All other kinds provide contradictory 
results.

In the parallel perfect fluid with the self-similarity of the first kind, we obtain a vacuum solution. The second kind leads to contradiction for EOS(1) but for $\operatorname{EOS}(2)$, we obtain a solution in which one fluid represents dust and the other vacuum. For $\operatorname{EOS}(3)$, we obtain a solution with arbitrary $\mu$ and $\alpha \neq \frac{3}{2}$. The zeroth kind yields a vacuum solution. There are three self-similar solution with self-similarity of the infinite kind one of which is Minkowski spacetime. For the parallel dust case, the first kind gives a contradiction. The second kind implies the same solution as in the parallel perfect fluid case with $\operatorname{EOS}(3)$ and $p_{2}=0, \alpha=\frac{3}{2}$. We do not have any self-similar solution of zeroth kind in the dust case. However, we obtain three different vacuum solutions for the infinite kind.

It is interesting to note that all the self-similar solutions, except the solutions given by Eqs.(33), (80), (97), (99), (104), (111), (165), (167), (170), (172) found here correspond with the already classified solutions [23] under particular coordinate transformations. The metrics given by Eqs.(52), (55), (74), (79) and (102) correspond to the class of metrics

$$
d s^{2}=d t^{2}-e^{2 \mu(t)} d x^{2}-e^{2 \lambda(t)}\left(d y^{2}+d z^{2}\right) .
$$

This metric has the four KVs admitting $G_{3} \otimes \Re$ with a spacelike $\Re$ and can be matched with Kantowski Sachs spacetimes [22]. The spacetime given by Eq.(57) correspond to the class of metrics

$$
d s^{2}=e^{2 \nu(x)} d t^{2}-d x^{2}-e^{2 \lambda(x)}\left(d y^{2}+d z^{2}\right)
$$

which has four KVs with the same Lie algebra and a timelike $\Re$. The solution given by Eq.(113) can be matched with the solution

$$
d s^{2}=e^{2 f(x)}\left[d t^{2}-e^{2 t / a}\left(d y^{2}+d z^{2}\right)\right]-d x^{2}, \quad(a \neq 0)
$$

which admits six KVs. The metrics (121) and (138) turn out to be equivalent to the metric

$$
d s^{2}=d t^{2}-e^{2 f(t)}\left[d x^{2}+e^{2 x / a}\left(d y^{2}+d z^{2}\right)\right], \quad(a \neq 0)
$$

which has six KVs with a Lie algebra identical to that of the metric (175) and belongs to the family of LRS metrics. Finally, the metrics given by Eqs.(77), (141), (158) and (168) has the correspondence with the class of metrics given as

$$
d s^{2}=d t^{2}-e^{\lambda(t)}\left(d x^{2}+d y^{2}+d z^{2}\right)
$$


admitting six KVs and represents FRW models. We also notice that the solutions given by the metrics (99), (104) seem to have the similar nature while the solutions (165) and (167), and solutions (170) and (172) can correspond to each other. It is worth mentioning that we obtain density either zero or positive in all the solutions except the one where it is not constant but can be positive. The physical properties of such solutions can be seen in [20]. Thus we finally obtain sixteen independent KSS plane symmetric solutions. The results can be summarized in the form of tables 1-6:

Table 1. Tilted Perfect Fluid KSS Solutions.

\begin{tabular}{|l|l|}
\hline Self-Similarity & Solution \\
\hline First kind (EOS(3)) & solution given by Eq.(33) \\
\hline Second kind (EOS(1)) & solution given by Eq.(52) \\
\hline Second kind (EOS(2)) & solution given by Eq.(52) \\
\hline Second kind (EOS(3))(i) & solution given by Eq.(52) \\
\hline Second kind (EOS(3))(ii) & solution given by Eq.(55) \\
\hline Second kind (EOS(3))(iii) & solution given by Eq.(57) \\
\hline Zeroth kind (EOS(1)) & solution given by Eq.(74) \\
\hline Zeroth kind (EOS(2)) & solution given by Eq.(74) \\
\hline Zeroth kind (EOS(3))(i) & solution given by Eq.(77) \\
\hline Zeroth kind (EOS(3))(ii) & solution given by Eq.(79) \\
\hline Zeroth kind (EOS(3))(iii) & solution given by Eq.(80) \\
\hline Infinite kind (EOS(1))(i) & Minkowski spacetime \\
\hline Infinite kind (EOS(1))(ii) & solution given by Eq.(97) \\
\hline Infinite kind (EOS(1))(iii) & solution given by Eq.(99) \\
\hline Infinite kind (EOS(2)) & Same solutions as in EOS(1) \\
\hline Infinite kind (EOS(3)) & Same solutions as in EOS(1) \\
\hline
\end{tabular}


Table 2. Tilted Dust KSS Solutions.

\begin{tabular}{|l|l|}
\hline Self-similarity & Solution \\
\hline First kind & None \\
\hline Second kind(i) & solution given by Eq.(102) \\
\hline Second kind(ii) & solution given by Eq.(52) \\
\hline Zeroth kind & None \\
\hline Infinite kind (i) & solution given by Eq.(104) \\
\hline Infinite kind (ii) & Minkowski spacetime \\
\hline Infinite kind (iii) & solution given by Eq.(97) \\
\hline Infinite kind (iv) & solution given by Eq.(99) \\
\hline
\end{tabular}

Table 3. Orthogonal Perfect Fluid KSS Solutions.

\begin{tabular}{|l|l|}
\hline Self-Similarity & Solution \\
\hline First kind & solution given by Eq.(111) \\
\hline Second kind & None \\
\hline Zeroth kind & None \\
\hline Infinite kind & Minkowski spacetime \\
\hline
\end{tabular}

Table 4. Orthogonal Dust KSS Solutions.

\begin{tabular}{|l|l|}
\hline Self-similarity & Solution \\
\hline First kind & solution given by Eq.(113) \\
\hline Second kind & None \\
\hline Zeroth kind & None \\
\hline Infinite kind & None \\
\hline
\end{tabular}


Table 5. Parallel Perfect Fluid KSS Solutions.

\begin{tabular}{|l|l|}
\hline Self-similarity & Solution \\
\hline First kind & solution given by Eq.(121) \\
\hline Second kind (EOS(1)) & None \\
\hline Second kind (EOS(2)) & solution given by Eq.(138) \\
\hline Second kind (EOS(3)) & solution given by Eq.(141) \\
\hline Zeroth kind (EOS(1)) & solution given by Eq.(158) \\
\hline Zeroth kind (EOS(2)) & solution given by Eq.(158) \\
\hline Zeroth kind (EOS(3)) & solution given by Eq.(158) \\
\hline Infinite kind (i) & solution given by Eq.(165) \\
\hline Infinite kind (ii) & Minkowski spacetime \\
\hline Infinite kind (iii) & solution given by Eq.(167) \\
\hline Infinite kind (iv) & Minkowski spacetime \\
\hline
\end{tabular}

Table 6. Parallel Dust KSS Solutions.

\begin{tabular}{|l|l|}
\hline Self-similarity & Solution \\
\hline First kind & None \\
\hline Second kind & solution given by Eq.(168) \\
\hline Zeroth kind & None \\
\hline Infinite kind (i) & Minkowski spacetime \\
\hline Infinite kind (ii) & Minkowski spacetime \\
\hline Infinite kind (iii) & solution given by Eq.(170) \\
\hline Infinite kind (iv) & Minkowski spacetime \\
\hline Infinite kind (v) & solution given by Eq.(172) \\
\hline
\end{tabular}

Finally, we would like to mention that Sintes et al. [14] found solutions only for the infinite kind. However, we have studied KSS solutions of the most general plane symmetric spacetimes in all kinds. The KSS solutions of the infinite kind can be matched with those of [14]. The solutions given by Eqs.(99) and (104) can be matched with Eq.(5.5) and the solutions (97), (170) and (172) correspond to the solution (5.6) of [14]. The remaining solutions do not correspond to those of the solutions given in [14].

\section{Acknowledgment}

One of us (SA) would like to thank HEC for Merit Scholarship. 


\section{References}

[1] Carr, B.J.: Phys. Rev. D62(2000)044022.

[2] Carr, B.J. and Coley, A.A.: Phys. Rev. D62(2000)044023.

[3] Maeda, H., Harada, T., Iguchi, H. and Okuyama, N.: Phys. Rev. D66(2002)027501.

[4] Maeda, H., Harada, T., Iguchi, H. and Okuyama, N.: Prog. Theor. Phys. 108(2002)819.

[5] Maeda, H., Harada, T., Iguchi, H. and Okuyama, N.: Prog. Theor. Phys. 110(2003)25.

[6] Sharif, M.: J. Math. Phys. 44(2003)5141; ibid 45(2004)1518; ibid 45(2004)1532.

[7] Sharif, M. and Aziz, Sehar: Int. J. Mod. Phys. D14(2005)1527;

Kinematic Self-Similar Solutions: Proceedings of the 11th Regional Conference on Mathematical Physics and IPM Spring Conference TehranIran, May, 3-6, 2004, eds. Rahvar, S., Sadooghi, N. and Shojai, F. (World Scientific, 2005)111.

[8] Cahill, M.E. and Taub, A.H.: Commun. Math. Phys. 21(1971)1.

[9] Carter, B. and Henriksen, R.N.: Annales De Physique 14(1989)47.

[10] Carter, B. and Henriksen, R.N.: J. Math. Phys. 32(1991)2580.

[11] Coley, A.A.: Class. Quant. Grav. 14(1997)87.

[12] McIntosh, C.B.G.: Gen. Relat. Gravit. 7(1975)199.

[13] Benoit, P.M. and Coley, A.A.: Class. Quant. Grav. 15(1998)2397.

[14] Sintes, A.M., Benoit, P.M. and Coley, A.A.: Gen. Relat. Gravit. 33(2001)1863.

[15] Carr, B.J., Coley, A.A., Golaith, M., Nilsson, U.S. and Uggla, C.: Class. Quant. Grav. 18(2001)303. 
[16] Carr, B.J., Coley, A.A., Golaith, M., Nilsson, U.S. and Uggla, C.: Phys. Rev. D61(2000)081502.

[17] Coley, A.A. and Golaith, M.: Class. Quant. Grav. 17(2000)2557.

[18] Sharif, M. and Aziz, Sehar: Int. J. Mod. Phys. D14(2005)73.

[19] Sharif, M. and Aziz, Sehar: Int. J. Mod. Phys. A20(2005)7579.

[20] Sharif, M. and Aziz, Sehar : J. Korean Physical Society 47(2005)757.

[21] Sharif, M. and Aziz, Sehar: J. Korean Physical Society 49(2006)21.

[22] Stephani, H., Kramer, D., Maccallum, M., Hoenselaers, C. and Herlt, E. Exact Solutions of Einstein's Field Equations (Cambridge University Press, 2003).

[23] Feroze, Tooba, Qadir, A. and Ziad, M.: J. Math. Phys. 42(2001)4947. 